\title{
Experimental Pig Model of Old Myocardial Infarction with Long Survival Leading to Chronic Left Ventricular Dysfunction and Remodeling as Evaluated by PET
}

\author{
Noboru Teramoto ${ }^{1}$, Kazuhiro Koshino ${ }^{1}$, Ikuo Yokoyama ${ }^{2,3}$, Shigeru Miyagawa ${ }^{4}$, Tsutomu Zeniya ${ }^{1}$, Yoshiyuki Hirano ${ }^{1}$, \\ Hajime Fukuda ${ }^{1}$, Junichiro Enmi ${ }^{1}$, Yoshiki Sawa ${ }^{4}$, Juhani Knuuti ${ }^{5}$, and Hidehiro Iida ${ }^{1}$ \\ ${ }^{1}$ Department of Investigative Radiology, National Cerebral and Cardiovascular Center Research Institute, Osaka, Japan; ${ }^{2}$ School of \\ Medicine and Faculty of Medicine, University of Tokyo, Tokyo, Japan; ${ }^{3}$ Department of Cardiovascular Medicine, Sanno Hospital, \\ International University of Health and Welfare, Tokyo, Japan; ${ }^{4}$ Department of Cardiac Surgery, Osaka University, School of \\ Medicine, Osaka, Japan; and ${ }^{5}$ Turku PET Centre, University of Turku, Turku, Finland
}

\begin{abstract}
A pig model of reduced left ventricular (LV) function and remodeling or chronic heart failure with long survival after myocardial infarction (MI) has not been established. The aim of this study was to evaluate the pathophysiologic status of a pig model of old MI using a series of PET studies. Methods: Twenty-seven male farm pigs were divided into 2 groups: 7 animals in the control group and 20 animals that underwent a proximal coronary artery (CA) occlusion using an ameroid constrictor after distal CA ligation. A series of PET examinations was performed to assess LV volumes, LV functions, myocardial perfusion response to adenosine, and viability as water-perfusable tissue index. Results: The distal CA ligation inhibited arrhythmia during and after the operation, and a transmural anteroseptal $\mathrm{MI}$, with an infarction area of $27 \% \pm 5 \%$ of the whole left ventricle, was generated with a survival rate of $75 \%$ at 4 mo. Wall motion evaluated by ${ }^{18}$ F-FDG PET was diffusely reduced, including the noninfarcted wall. Global LV ejection fraction as assessed by gated $\mathrm{C}^{15} \mathrm{O}$ PET was reduced $(39 \% \pm$ $16 \%)$ in the group undergoing occlusion, compared with the control group $(66 \% \pm 16 \%, P<0.05)$. LV end-systolic $(31.4 \pm 9.2$ $\left.\mathrm{cm}^{3}\right)$ and end-diastolic (52.7 $\left.\pm 10.2 \mathrm{~cm}^{3}\right)$ volumes were increased, compared with controls $\left(15.2 \pm 9.4 \mathrm{~cm}^{3}, P<0.01\right.$, and $41.7 \pm 11.5 \mathrm{~cm}^{3}, P<0.05$, respectively). Histology showed hypertrophy and development of microscopic fibrosis in noninfarcted myocardium. PET demonstrated the reduced myocardial perfusion response to adenosine and also reduced water-perfusable tissue index in remote segments. Conclusion: The pig model of old Ml generated by the chronic proximal CA obstruction after distal ligation was characterized by LV dysfunction and remodeling, with a high survival rate.
\end{abstract}

Key Words: experimental model; PET; myocardial flow reserve; remodeling; regeneration therapy

J Nucl Med 2011; 52:761-768

DOI: 10.2967/jnumed.110.084848

Received Oct. 30, 2010; revision accepted Dec. 9, 2010.

For correspondence or reprints contact: Hidehiro lida, Department of Investigative Radiology, National Cardiovascular Center Research Institute,

5-7-1 Fujishiro-dai, Suita City, Osaka, Japan 565-8565.

E-mail: iida@ri.ncvc.go.jp

COPYRIGHT $\odot 2011$ by the Society of Nuclear Medicine, Inc.
C cern (1). Myocardial infarction (MI) is the cause of CHF in two thirds of the patients, and the morbidity and mortality remain high $(2,3)$. The potential therapies, such as new class of pharmacologic agents and cell therapy (4), need to be tested in proper animal models to demonstrate the effects and outcome before initiating clinical trials. Dogs have been extensively used in heart research. Because the coronary arterial systems in dogs can develop collaterals quickly when myocardial ischemia occurs, it has been difficult to produce a large MI that typically introduces $\mathrm{CHF}$ with general characteristics of left ventricular (LV) remodeling (5).

Pigs have been considered better suited than dogs for pathophysiologic research of ischemic heart diseases, because the coronary system of pigs is more similar to that of humans (6). Tolerance of ischemia and denervation after ischemia in pigs is also similar to that in humans (6). Because of the delayed development of collaterals after occlusion, ligation of a peripheral part of the coronary arterial system generates a small MI (7). However, an experimental model of large MI introducing global LV dysfunction is difficult to develop, because sudden cardiac death (SCD) due to fatal arrhythmias and an intolerance of ischemia frequently occurs in pigs (8). The models of small MI made by the ligation of a peripheral part of the coronary arterial system demonstrate reasonably good survival rates but only for a small infarction. The model of small MI using a coronary ameroid constrictor (model MRI-2.50-TI; Research Instruments SW) has also demonstrated moderate SCD rates (6,8-13-15), but the animals develop primarily chronic ischemia or hibernating myocardium, without a significant amount of scar tissue. Thus, the limitations of current models are that the infarcted region is small and that the hearts are not developing a clinical picture of CHF with global LV dysfunction, LV dilatation, and remodeling.

On the other hand, Shen et al. (16) developed an experimental pig model of MI and heart failure. Sequential 
ligation of distal and proximal coronary arteries was used to establish MI with a reasonable survival rate, and pacing tachycardia was used to achieve heart failure. However, this pig model was studied over only a short term $(21 \mathrm{~d})$ and required pacing tachycardia to cause CHF. There has been little evaluation of pathophysiology and no evidence of the presence of cardiac remodeling.

This aim of this study was to characterize the pig model of old MI. We first reinvestigated the technique of generating a pig model of relatively large MI causing global LV dysfunction and LV remodeling in segments remote from the infarcted region, by means of the sequential ligation of distal and proximal coronary arteries without the pacing procedures. We then evaluated the pathophysiologic characteristics of this animal model by comprehensively analyzing histology, LV volumes and LV function, myocardial perfusion response to adenosine, and perfusable tissue fractions (PTF) in the remote segment, using an advanced PET technique.

\section{MATERIALS AND METHODS}

\section{Subjects}

Male farm pigs, 3 mo old at the start of the study (weight range, $18-23 \mathrm{~kg}$; mean weight $\pm \mathrm{SD}, 20 \pm 1.2 \mathrm{~kg}$ ), were used. The animals were divided into 2 groups. Group A consisted of 7 pigs without any operation and was designated as the control group (average body weight, $26 \pm 2.4 \mathrm{~kg}$ ). Group B consisted of 20 pigs that underwent occlusion using an ameroid constrictor (7); ligation of the distal left anterior descending coronary artery (LAD) was performed before the ameroid constrictor was used. Of the 16 pigs that survived for 4 mo after the operation, 7 underwent PET studies. The other 9 were assigned to tissue-regenerative projects. The average body weight of this group of 7 pigs was $42 \pm 8.2 \mathrm{~kg}$ at the time of the PET scan.

Animals were maintained and handled in accordance with guidelines for animal research (17). The study protocol was approved by the local Committee for Laboratory Animal Welfare, National Cardiovascular Center, Osaka, Japan.

\section{Permanent Occlusion Procedures}

In group $\mathrm{B}$, permanent occlusion was made at the proximal LAD using an ameroid constrictor (18) (Fig. 1) as follows. Pigs were preanesthetized by an intramuscular injection of ketamine hydrochloride $(20 \mathrm{mg} / \mathrm{kg}$; Ketaral [Diichi-Sankyo]) and xylazine hydrochloride (2 mg/kg; Celactal [Bayer HealthCare]). The animals were positioned supine, and a 22-gauge indwelling needle (Surflo F\&F; Terumo) was inserted in the central vein of the auricle. A 3-way cock (Terufusion TS-TR2K; Terumo) was attached to the external cylinder of the indwelling needle and connected for continuous anesthetic injection. The animals were intubated with an endotracheal cannula (6 French; Sheridan) and then connected to an artificial respirator (Single Animal Volume Controlled Ventilators model 613 [Harvard Apparatus]) with a stroke volume of $200-300 \mathrm{~cm}^{3} /$ stroke and frequency of $20 / \mathrm{min}$. Propofol (6 mg/kg/h; Diprivan [Astra-Zeneca]) and vecuronium bromide $(0.05 \mathrm{mg} / \mathrm{kg} / \mathrm{h}$; Musculux [Sankyo Yell Yakuhin Co., Ltd.]) were continuously infused using a syringe pump (Terufusion TE-3310N; Terumo). Then, the animals were fixed in a recumbent position so that the left thorax was exposed, and the outer layer of skin and muscles between the third and fourth ribs was dissected. The distance between the third and fourth ribs was widened with a rib spreader to allow a direct view of the left auricle and LAD. The pericardium was dissected along the $\mathrm{LAD}$, from the upper part of the left auricle $(\sim 6 \mathrm{~cm})$, to expose the myocardium around the LAD. The LAD on the proximal side, below the left auricle from the myocardium, was exfoliated for approximately $1 \mathrm{~cm}$. A lidocaine hydrochloride jelly (Xylocaine jelly; Astra-Zeneca) was applied to anesthetize the area.

A complete ligation was first made on the distal LAD (no. 9), immediately after the second diagonal branch, using a suture (2-0; Nescosuture) approximately $20 \mathrm{~min}$ before the ameroid constrictor was fastened. An ameroid constrictor (COR-2.50-SS; Research Instruments) was then fastened using sutures as displayed in Figure 1 . To enhance the effect of the ameroid constrictor, 2 additional suture strings were loosely rounded at the site of the ameroid so that these strings were located between (below) the ameroid constrictor and arterial wall.

\section{PET Procedures}

After fasting overnight, the pigs were sedated with ketamine hydrochloride $(20 \mathrm{mg} / \mathrm{kg})$ and xylazine hydrochloride $(2 \mathrm{mg} / \mathrm{kg})$ by intramuscular injection. Anesthesia was induced and maintained with intravenous propofol $(6 \mathrm{mg} / \mathrm{kg} / \mathrm{h})$ and vecuronium bromide $(0.05 \mathrm{mg} / \mathrm{kg} / \mathrm{h})$. The animals were intubated and mechanically ventilated with a mixture of $25 \%$ oxygen and $75 \%$ nitrogen at $10 \mathrm{~mL} / \mathrm{kg}$ plus $50 \mathrm{~mL} / \mathrm{stroke}$ at 20 strokes/min. Catheters were placed in the femoral artery to monitor the arterial blood pressure and in the femoral vein to infuse $\mathrm{H}_{2}{ }^{15} \mathrm{O}$ or ${ }^{18} \mathrm{~F}$-labeled FDG. Systolic and diastolic blood pressure, heart rate, and arterial blood gases were monitored.

A series of PET scans was obtained using an ECAT-HR tomograph (CTI Inc.). The blood-pool images were obtained after the animals inhaled $2.7 \mathrm{GBq}$ of $\mathrm{C}^{15} \mathrm{O}$ gas (19). Arterial blood samples were taken every minute during the $\mathrm{C}^{15} \mathrm{O}$ scan, and their radioactivity concentration in the whole blood was measured. Additional electrocardiogram-gated $\mathrm{C}^{15} \mathrm{O}$ images were obtained (16 gates). After $12-15$ min of ${ }^{15} \mathrm{O}$ radioactivity decay, 7 dynamic $\mathrm{H}_{2}{ }^{15} \mathrm{O}$ PET scans were acquired at intervals of $12-15 \mathrm{~min}$. The first and the last scans were obtained without pharmacologic stress, and the second through sixth scans were obtained during intravenous infusion of adenosine (100, 200, 400, 600, and 800

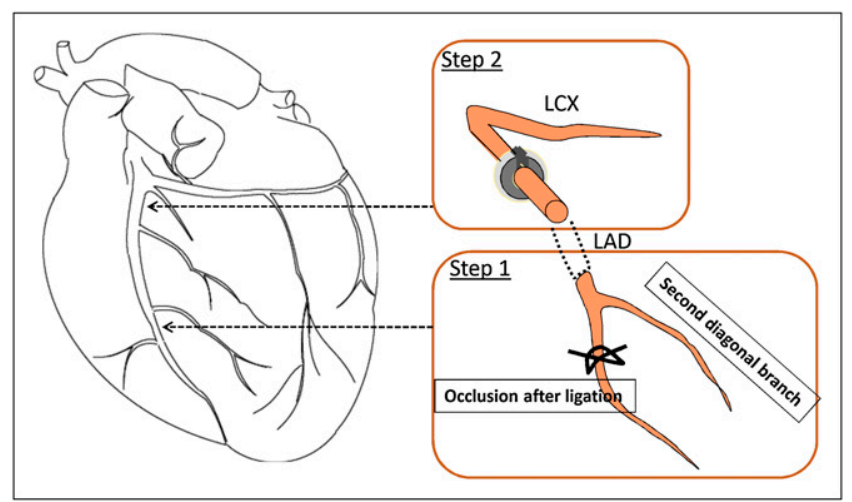

FIGURE 1. Ligation of LV LAD. Distal LAD after second diagonal branch was ligated, and $30 \mathrm{~min}$ later ameroid constrictor was placed at proximal LAD. Two suture strings were inserted between ameroid constrictor and arterial wall to make occlusion complete. LCX = left circumflex artery. 
[only for group A] $\mu \mathrm{g} / \mathrm{kg} / \mathrm{min}$ ). The 6-min dynamic scan of 26 frames $(12 \times 5,8 \times 15$, and $6 \times 30 \mathrm{~s})$ was started when radioactivity appeared in the right ventricle. Furthermore, another PET scan using ${ }^{18} \mathrm{~F}$-FDG was acquired using a euglycemic hyperinsulinemic clamp (20,21). For this study, insulin $(4 \mathrm{mIU} / \mathrm{kg} / \mathrm{h})$ and glucose $(5-8 \mathrm{mg} / \mathrm{kg} / \mathrm{min})$ were continuously infused over $2 \mathrm{~h}$, and then approximately $111 \mathrm{MBq}$ of ${ }^{18} \mathrm{~F}-\mathrm{FDG}$ was intravenously injected. The blood glucose was $109 \pm 10.6,105 \pm 3.8$, and $107 \pm 10.0 \mathrm{mg} / \mathrm{dL}$ at the time of ${ }^{18} \mathrm{~F}-\mathrm{FDG}$ injection. PET images were acquired dynamically for $60 \mathrm{~min}$, and the images acquired during the last $30 \mathrm{~min}$ were used for further analysis. An additional electrocardiogram-gated scan was obtained for $30 \mathrm{~min}$ to estimate myocardial wall motion and wall thickening.

After the PET scans, coronary angiography was undertaken to confirm the occlusion of LAD. A portable angiographic camera (Digital Mobile Imaging System OEC Series 9800; GE Healthcare U.K. Ltd.) was used with the contrast medium (Omnipaque 300; Daiichi-Sankyo).

\section{Histologic Analysis}

At the end of the study, pentobarbital sodium was administered, and animals were euthanized. The animals' hearts were then excised and sliced at a thickness of $10 \mathrm{~mm}$ to correspond to PET images (22). The slices were stained with Masson trichrome and hematoxylin and eosin. The surface area of the infarcted LV wall on the stained slices was calculated according to a previously describe procedure (22). The length of the infarcted zone, including both transmural and nontransmural infarction, was measured on each of the stained slices along the LV wall. The defect surface area was then calculated as a summation of the defect length multiplied by the slice interval. The cell and microvascular structures were also analyzed. In particular, development of fibrosis, cell enlargement, and degeneration of the vascular network were visually estimated. The analysis was masked from the other analyses.

\section{Data Analysis}

The extravascular tissue density images $(\mathrm{g} / \mathrm{mL})$ were calculated by subtracting the $\mathrm{C}^{15} \mathrm{O}$ blood volume from the transmission images (23). The relative images of myocardial blood flow (MBF) and water-PTF $(\mathrm{g} / \mathrm{mL})$ were also generated from the dynamic $\mathrm{H}_{2}{ }^{15} \mathrm{O}$ images $(22,23)$. The relative accumulation of ${ }^{18} \mathrm{~F}-\mathrm{FDG}$ was calculated using validated techniques (24). The regions of interest were placed in the anterior wall and lateralanterior wall, which corresponded to the MI and normal-tissue regions, respectively. The regions of interest were then copied to other images, and the quantitative parameters of regional $\mathrm{MBF}$ $(\mathrm{mL} / \mathrm{min} / \mathrm{g})$, PTF $(\mathrm{g} / \mathrm{mL})$, and arterial blood volume $\mathrm{V}_{\mathrm{a}}(\mathrm{mL} /$ $\mathrm{mL}$ ) were calculated using nonlinear least-squares fitting as described previously $(22,23)$. These calculations were done for all $\mathrm{H}_{2}{ }^{15} \mathrm{O}$ PET studies to estimate baseline MBF and the adenosine-based responses both in MI and in non-MI regions.

The water-perfusable tissue index (PTI) $(23,25,26)$, which was defined as the fraction of water-perfusable tissue over total tissue, was calculated by dividing PTF by the extravascular tissue density images. This calculation was done for each myocardial region and compared between the infarcted anterior wall and normal posterior-lateral wall regions. The obtained values were also compared with control subjects.

The LV ejection fraction and cardiac output were evaluated by counting the total counts within the LV area from the typical electrocardiogram-gated $\mathrm{C}^{15} \mathrm{O}$ images shown in Figure 2. The wall motion was analyzed using electrocardiogram-gated ${ }^{18} \mathrm{~F}-\mathrm{FDG}$ images. The wall motion score was divided into 4 levels (normal, 0; hypokinetic, 1; akinetic, 2; and dyskinetic, 3) and defined for the anterior wall and contralateral (lateral-posterior) wall regions.

The surface area of the infarcted LV wall on the ${ }^{18} \mathrm{~F}-\mathrm{FDG}$ and PTF images was calculated as described previously (22). A significant defect was defined as a value less than $50 \%$ of that in the control region, which was defined in the contralateral region, indicating preserved ${ }^{18} \mathrm{~F}-\mathrm{FDG}$, preserved PTF, and elevated MBF during adenosine. The myocardial midlines were then traced along the $50 \%$ of peak count boundary of the $\mathrm{C}^{15} \mathrm{O}$ blood volume images at each slice (22). The surface area of the defect was calculated as a summation of the defect length multiplied by the slice interval. These defect surface areas were compared with those obtained from the stained slices.

All data were presented as mean \pm SD. Pearson correlation and linear regression analyses were used to evaluate relationships between the 2 values. A $P$ value of less than 0.05 was considered statistically significant.

\section{RESULTS}

\section{Survival Rates of Animal Groups}

No adverse events were detected in group A; in group B, 4 pigs (20\%) died within a month and an additional pig 1 mo after that. Thus, the total survival rate at 4 mo was $75 \%$ in group B. Severe arrhythmia occurred in all animals in group B during the operation. The arrhythmia and fibrillation, introducing the sudden cardiac death, were significantly suppressed at approximately $20 \mathrm{~min}$ after ligation of the distal LAD. Figure 3 displays the survival rates of the pigs that underwent the operation.

Of the pigs in group B that survived for $4 \mathrm{mo}, 7$ were selected for further characterization and imaging studies. These 7 animals were compared against the animals in group A, on which no operation was performed. The characteristics of the 2 groups at the time of the imaging

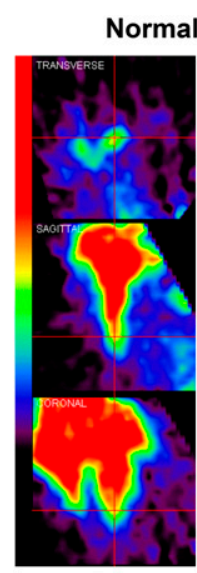

Systolic

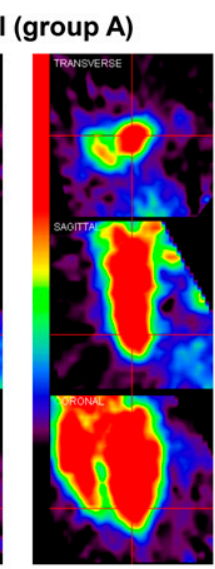

Diastolic

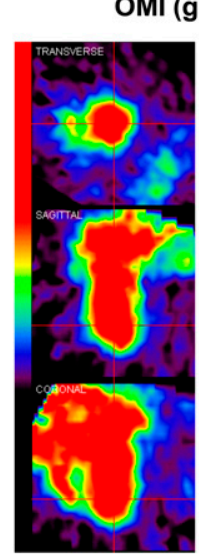

Systolic
MI (group B)

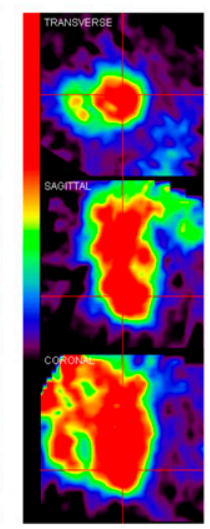

Diastolic
FIGURE 2. Typical images obtained from electrocardiogram-gated PET scans with $\mathrm{C}^{15} \mathrm{O}$ inhalation at end-systolic and end-diastolic phases. $\mathrm{OMI}=$ old $\mathrm{MI}$. 


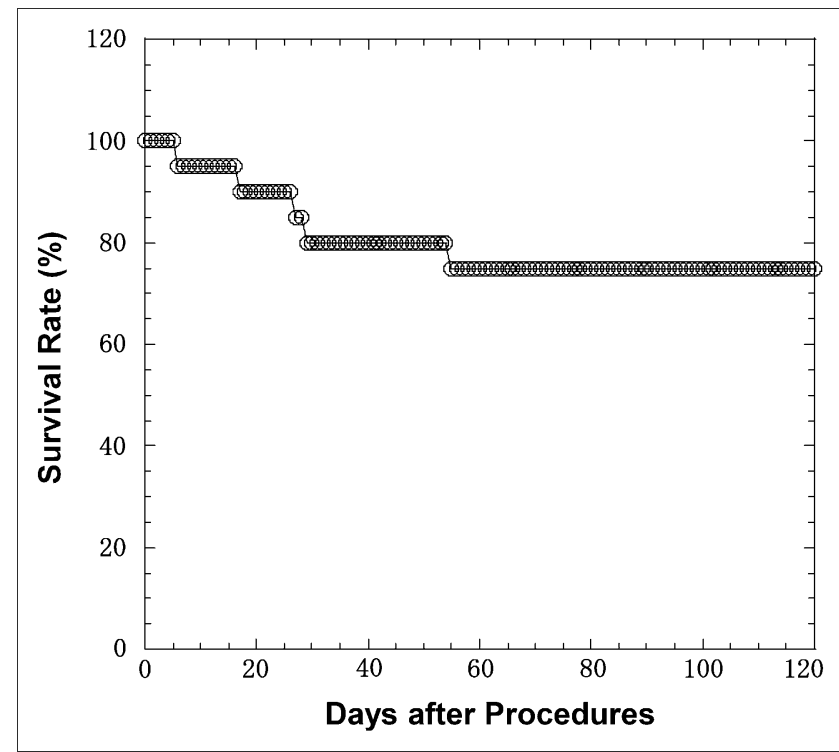

FIGURE 3. Survival rates for group B after ameroid constrictor was placed.

studies are displayed in Table 1. Because the animals in group B were allowed to grow after operation, they were larger at the time of the imaging studies.

\section{Characteristics of Groups}

Table 1 shows the hemodynamic data for the animals in groups A and B. Compared with group A, group B showed a significantly reduced heart rate and diastolic blood pressure and, thus, rate-pressure product. In addition, the global $\mathrm{LV}$ ejection fraction measured with gated $\mathrm{C}^{15} \mathrm{O}$ PET in group B was significantly reduced $(39 \% \pm 16 \%$ [24\%$59 \%$ ] in group B vs. $66 \% \pm 16 \%$ [46\%-91\%] in group A, $P<0.01)$. LV end-systolic volume in group $\mathrm{B}$ was significantly increased $\left(31.4 \pm 9.2 \mathrm{~cm}^{3}\right.$ in group B vs. $15.2 \pm 9.4 \mathrm{~cm}^{3}$ in group A, $P<0.01$ ), and LV end-diastolic volume was also significantly increased $\left(52.7 \pm 10.2 \mathrm{~cm}^{3}\right.$ in group B vs. $41.7 \pm 11.5 \mathrm{~cm}^{3}$ in group A, $P<0.05$ ). Increased end-systolic and end-diastolic volumes are also clearly visible in Figure 2. Systolic blood pressure and diastolic blood pressure, as well as rate-pressure product, were reduced during adenosine study in both groups. Heart rate was increased in group A but not in group B. Other parameters such as hemoglobin concentration $(\mathrm{g} / \mathrm{dL})$, percentage saturation of arterial $\mathrm{O}_{2}, \mathrm{PaCO}_{2}(\mathrm{~mm} \mathrm{Hg})$, and $\mathrm{PO}_{2}$ $(\mathrm{mm} \mathrm{Hg})$ were not significantly different between the 2 groups. Table 2 shows detailed hemodynamic parameters and the results from the histologic analysis for each individual animal in group B. Wall motion score was reduced (akinesis-dyskinesis or 2-3) in the anterior wall, which includes the area of MI. The wall motion score was also reduced diffusely in all animals and indicated hypokinesis (grade 1) in the infarct-remote posterolateral wall. The coronary angiography demonstrated complete occlusion of the LAD in all animals in group B. As shown in Figure 4, no clear collateral circulation existed.

\section{Imaging Results}

The myocardial perfusion $\left(\mathrm{H}_{2}{ }^{15} \mathrm{O}\right)$ and metabolism $\left({ }^{18} \mathrm{~F}-\right.$ FDG) images at the mid ventricular plane and their corresponding slices after histochemical staining for the animals in group B are displayed in Figure 5. In all animals, clear signs of MI were detected in the anterior wall. The surface area of the MI was $13.7 \pm 4.3 \mathrm{~cm}^{2}$, which corresponded to $27 \% \pm 9 \%$ of the whole left ventricle. Both PTF and ${ }^{18} \mathrm{~F}-$ FDG images visually agreed well with the stained slices. Moreover, the ${ }^{18} \mathrm{~F}-\mathrm{FDG}$ images agreed well with the qualitative PTF images in all animals. The spatial distribution and size of the developed MI are visually reproducible

TABLE 1

Physiologic Parameters of Pigs at Time of PET

\begin{tabular}{|c|c|c|}
\hline Parameter & Group A (control, $n=7$ ) & Group B (old MI, $n=7$ ) \\
\hline Body weight $(\mathrm{kg})$ & $26 \pm 2.4$ & $41 \pm 8.2$ \\
\hline Heart rate (beats. $\min ^{-1}$ ) & $72 \pm 22.4\left(82 \pm 21.4^{\star}\right)$ & $52 \pm 6.3^{\dagger}(53 \pm 9.2)$ \\
\hline Systolic blood pressure $(\mathrm{mm} \mathrm{Hg})$ & $131 \pm 22.1\left(95 \pm 22.4^{\ddagger}\right)$ & $111 \pm 18.3^{\dagger}\left(97 \pm 15.0^{*}\right)$ \\
\hline Diastolic blood pressure $(\mathrm{mm} \mathrm{Hg})$ & $92 \pm 19.3\left(49 \pm 16.7^{\ddagger}\right)$ & $74 \pm 13.6^{\dagger}\left(57 \pm 11.9^{\ddagger}\right)$ \\
\hline Rate pressure product $\left(\mathrm{mm} \mathrm{Hg} \cdot \mathrm{min}^{-1}\right)$ & $9,567 \pm 3,616\left(5,657 \pm 3201^{\ddagger}\right)$ & $5,732 \pm 1,151^{\dagger}\left(4,445 \pm 948^{\star}\right)$ \\
\hline Wall thickness (posterior-lateral wall) (cm) & $1.2 \pm 0.1$ & $1.6 \pm 0.1^{\ddagger}$ \\
\hline End-diastolic LV volume $(\mathrm{mL})$ & $41.7 \pm 11.5$ & $52.73 \pm 10.2^{\dagger}$ \\
\hline End-systolic LV volume (mL) & $15.2 \pm 9.4$ & $31.4 \pm 9.2^{\star}$ \\
\hline LV ejection fraction (\%) & $66.0 \pm 16.2$ & $39.7 \pm 16.9^{\star}$ \\
\hline Hemoglobin concentration $(\mathrm{g} / \mathrm{dL})$ & $13 \pm 1.5$ & $12 \pm 0.9$ \\
\hline Saturation of arterial $\mathrm{O}_{2}(\%)$ & $99 \pm 1.0$ & $99 \pm 0.6$ \\
\hline $\mathrm{pCO}_{2}(\mathrm{~mm} \mathrm{Hg})$ & $39 \pm 2.7$ & $41 \pm 2.8$ \\
\hline $\mathrm{pO}_{2}(\mathrm{~mm} \mathrm{Hg})$ & $125 \pm 20.1$ & $141 \pm 11.4$ \\
\hline $\begin{array}{l}{ }^{\star} P<0.01 \\
{ }^{\dagger} P<0.05 \\
{ }^{\ddagger} P<0.001 \\
\text { Data are mean } \pm \text { SD. Values in parenthes }\end{array}$ & ministration of maximum dose & \\
\hline
\end{tabular}




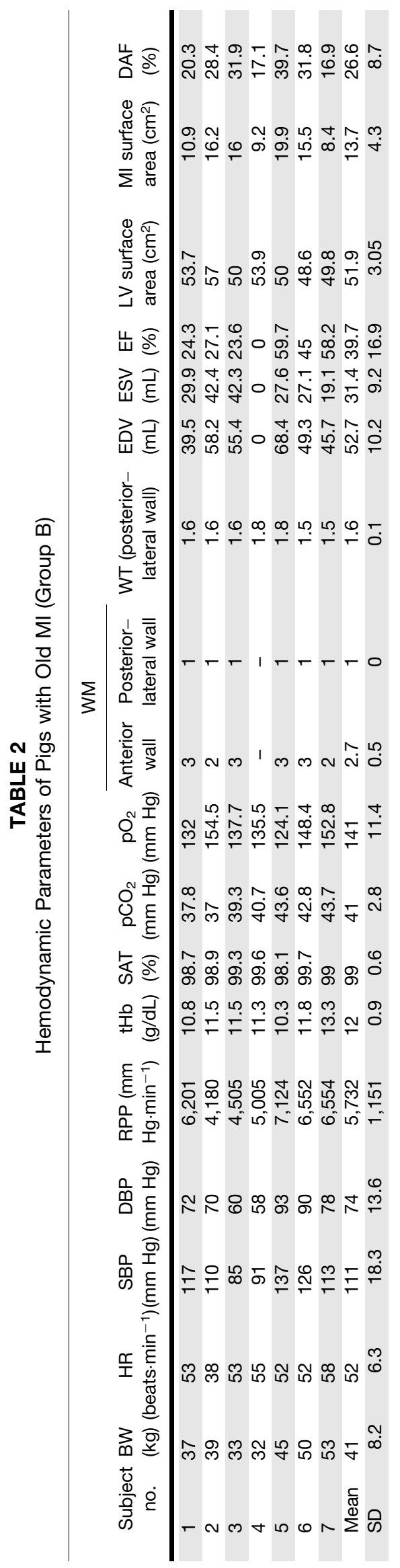

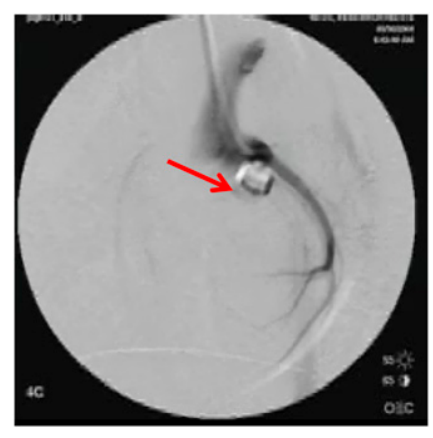

FIGURE 4. Example of coronary angiogram in animal with ameroid constrictor (arrow) (group B).

among the animals. Myocardial hypertrophy was visible in the wall regions remote from the infarcted area. In group B, the posterior-lateral wall thickness estimated from histochemical staining was $16 \pm 1 \mathrm{~mm}$ (Table 2), which was significantly greater than that of group A $(12 \pm 1 \mathrm{~mm}$; Table 1) $(P<0.001)$.

Further analysis of the size of the infarcted region is displayed in Figure 5B. We excluded 1 animal whose ${ }^{18} \mathrm{~F}-$ FDG PET images demonstrated high accumulation at the anterior LV area (and therefore a smaller value in the defect surface area), which was attributed to the adhesion or inflammation between the myocardial and chest walls. Thus, the defect area on histology showed intersubject variation of approximately $20 \%$. The results of both ${ }^{18} \mathrm{~F}-$ FDG and PTF image analysis and histochemical analysis agreed well (Fig. 5B). Baseline and adenosine-stimulated MBF were also clearly blunted in the anterior wall in the animals in group B (Fig. 6A). Interestingly, abnormally reduced adenosine flow response also was detected in the myocardial regions remote from the $\mathrm{MI}$ in group $\mathrm{B}$, whereas the baseline flow values were normal.

Further analysis of myocardial PTF and PTI values-indices of PTF-revealed that these values were significantly reduced in the infarcted anterior wall in group B (Fig. 6B). PTI was also moderately but significantly $(\sim 10 \%)$ reduced in the remote myocardial wall in group $\mathrm{B}$.

\section{Histologic Analysis}

Histologic analysis did not show any signs of MI in group A, but transmural anterior wall MI was apparent in all animals in group B. Hypertrophy was detected in the posterior wall of group B in all animals. Multinuclear muscle cell breeding (Fig. 7A) and hyperplasia of blood vessels (Fig. 7B) were also visible in all animals in group $\mathrm{B}$, mostly in the subendocardial regions. Denaturation necrosis with epicardial fibrous change and hypertrophy has also been seen in the lateral-posterior wall regions of group B (Fig. 7C).

\section{DISCUSSION}

This study demonstrated that $75 \%$ of pigs with old MI generated by the 2 steps of LAD ligation survived more than 4 mo. Such a long survival has never, to our 
FIGURE 5. (A) Histologic, PTF, and ${ }^{18} \mathrm{~F}-$ FDG slices at middle level of left ventricle in 7 animals of group B. Spatial distribution and size of $\mathrm{MI}$ are visually reproducible among animals. Myocardial hypertrophy was visible in remote myocardial wall regions. (B) Comparison of defect surface areas obtained from histologic stained slices with PET images using ${ }^{18} \mathrm{~F}-\mathrm{FDG}$ and PTF. MTC $=$ Masson trichrome.

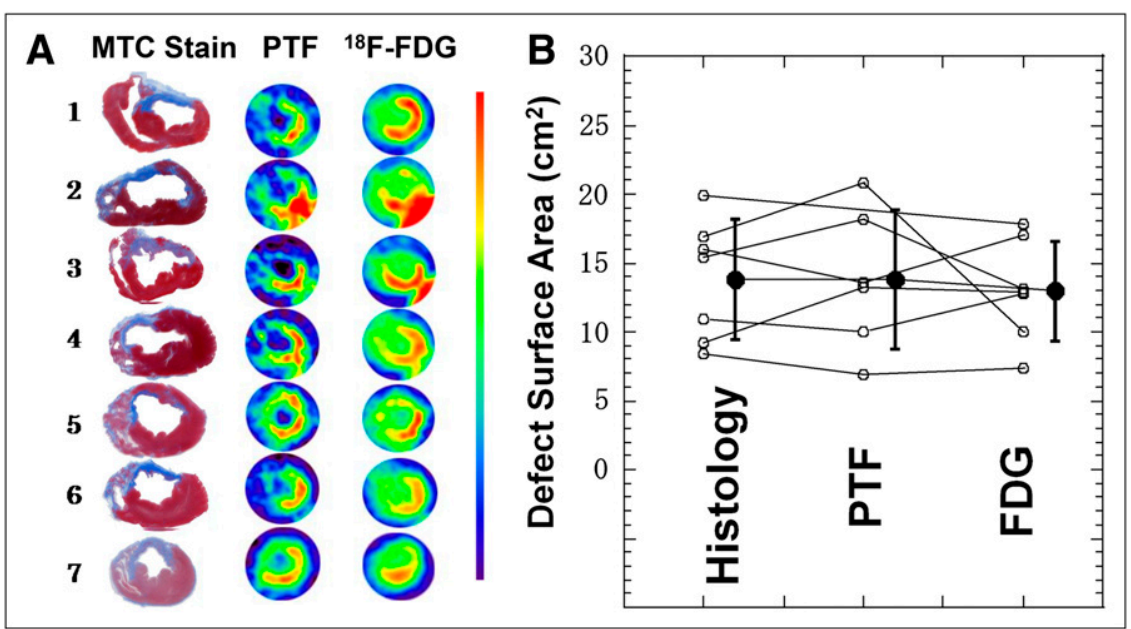

knowledge, been accomplished in any studies of pig heart disease. The size of MI reached approximately $27 \%$ of the whole left ventricle, causing the remodeling and global LV dilatation (significantly increased LV end-systolic and enddiastolic volumes) to be associated with reduced global LV function. PET with $\mathrm{C}^{15} \mathrm{O}$ and ${ }^{18} \mathrm{~F}-\mathrm{FDG}$ showed that regional LV wall motion was impaired not only in the infarcted region but also in the myocardial areas remote from the MI. PET also demonstrated reduced MBF reactivity in remote regions in addition to the infarct area. PTI was also reduced in the remote region, suggesting development of microscopic fibrosis. Moreover, other findings from histology indicated the existence of abnormalities in the noninfarcted area remote from the MI. These results indicate that this animal model may be close to human CHF after MI.

Shen et al. (16) adopted additional pacing tachycardia in pigs after sequential coronary artery ligations and observed global LV dysfunction, claiming that CHF was introduced. The present study is similar to the study by Shen et al. (16) but is based on only sequential coronary artery obstructions accomplished with acute distal coronary artery occlusion followed by a chronic proximal coronary occlusion with an ameroid constrictor, causing similar LV dysfunction. Of note was the better survival rate $(80 \%$ at $1 \mathrm{mo}, 75 \%$ at $4 \mathrm{mo}$ ) demonstrated in the current report than in any previous report $(6,8-12,14,16)$, including Shen et al. $(73.3 \%$ at $21 \mathrm{~d})$. In addition, it has not been confirmed whether such a good survival rate at $21 \mathrm{~d}$ could last more than 4 mo after producing global LV dysfunction with MI. Our preliminary experiment, independently performed on 69 farm pigs, demonstrated that when the proximal LAD was occluded by the ameroid constrictor alone, 45 pigs $(65 \%)$ died within a month, and the total survival rate was $30 \%$ at 4 mo. This survival rate is significantly smaller than the rates from the present study.

The reason why animals with MI acquired such good survival is not fully understood. However, it was clearly observed that the ligation of the peripheral coronary artery before the gradual occlusion of the main trunk of the LAD apparently inhibited the fibrillation during the operation. This procedure is obviously effective at protecting against
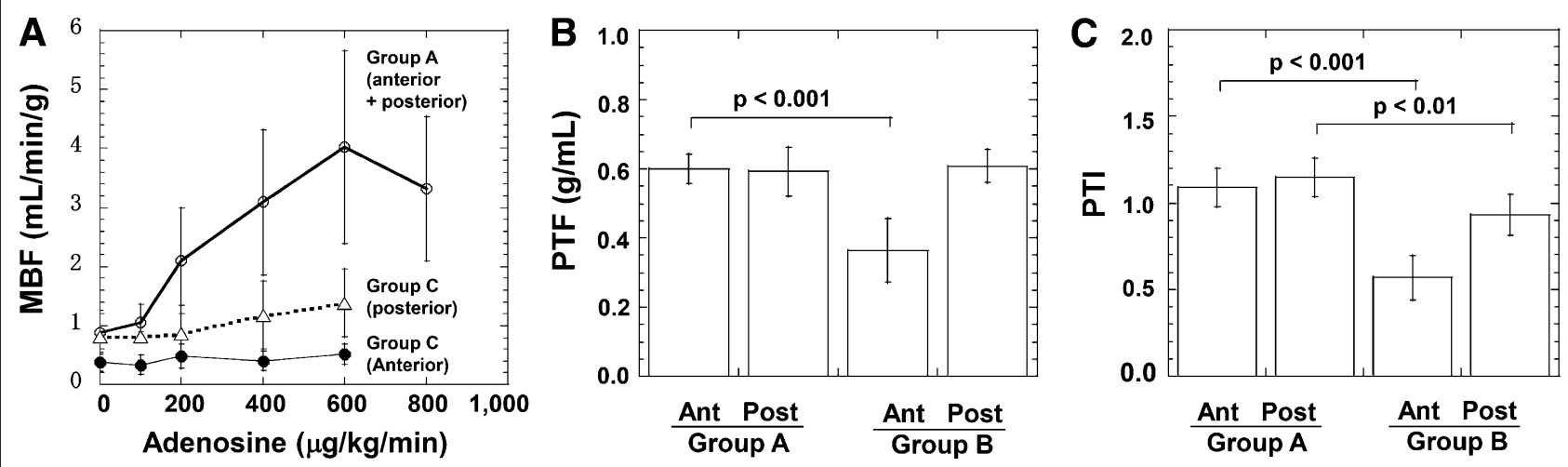

FIGURE 6. Results from PET studies. (A) Regional MBF as function of adenosine dose. In group A, MBF increases with increasing adenosine dose. In infarcted anterior wall of group B, MBF is reduced at rest and does not respond to adenosine. In posterior wall region, adenosine reactivity was significantly reduced. (B and C) Comparison of water-PTF (B) and water-PTI (C). Both were reduced in anterior wall of group B. PTI was also reduced in posterior wall in group B. Ant = anterior; Post $=$ posterior. 
A

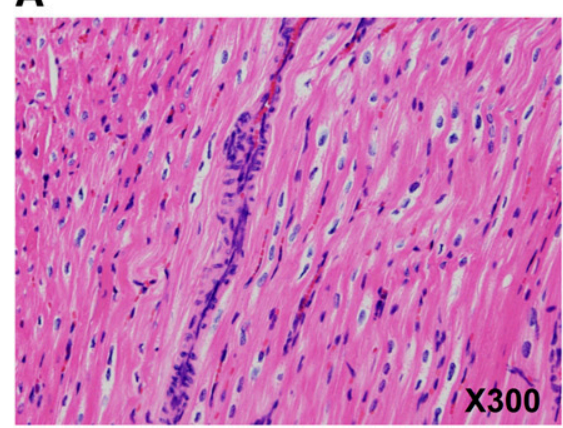

B

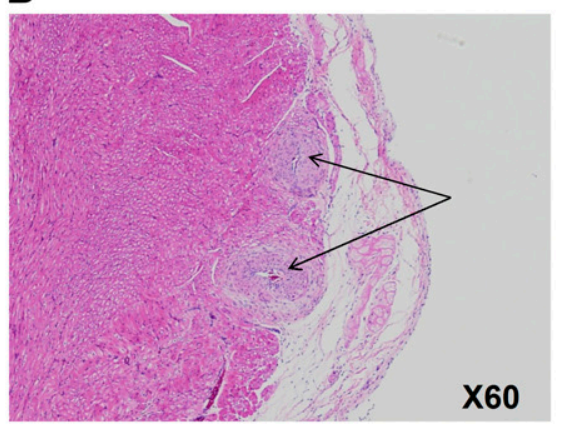

C

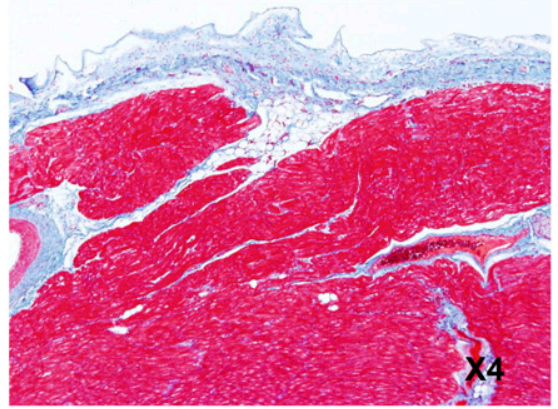

FIGURE 7. Typical images of histologic staining obtained from group B. Hypertrophy with multinuclear muscle cell breeding (A), hyperplasia of blood vessels shown as arrows (B), and denaturation necrosis often seen in subendocardial regions, as in this picture (C), are typical findings.

fatal arrhythmias. This preconditioning effect is supported by the experiments of Moses et al. (27), in which ischemic preconditioning of the distal coronary artery protected cardiac muscle through a mechanism involving the potassium channels of mitochondria and myocytes. There may be contributions from other preconditioning factors $(9,28-30)$ that associate with increased tolerance of ischemia. Another mechanism could be associated with the modulation of sympathetic tone. It has been reported that regional heterogeneity in myocardial cellular mechanisms (responsible for myocardial cellular depolarization) and repolarization in hibernating myocardium (12) can induce ventricular fibrillation (9). Inhibition of such heterogeneous myocardial cellular mechanisms or control of modulated sympathetic tone could be another possible explanation.

The present study used a gradual total occlusion of the proximal of LAD, as evidenced by the coronary angiography (Fig. 4), and thus caused an MI of approximately $27 \%$ of the total myocardium. The global LV ejection fraction in group B was significantly reduced (39\% vs. $66 \%$ in controls), showing that LV systolic function was impaired in group B. Both histology and PET had concordant findings. The baseline MBF in the MI area was reduced and was not responding to adenosine administration. This finding is reasonable because the infarcted tissue should have reduced capillary density, and the resistive vessels do not respond to any vasodilating stimulation. The physical extent and size of the MI were reproducible among the individual animals. This feature is important when this animal model is applied to evaluate various new pharmaceuticals or various regenerative therapeutic trials.

The noninfarcted myocardium developed clear signs of remodeling, such as hypokinesis, hypertrophy, and the accumulation of fibrotic tissue in the remote myocardial wall, and significantly decreased PTI values, a marker of residual fractionation of water-perfusable tissue. The baseline MBF was preserved, but reactivity to the adenosine was blunted, even at a maximum dose of $600 \mu \mathrm{g} / \mathrm{kg} / \mathrm{min}$. This reduced reactivity could be related to the degeneration of small arteries, possibly caused by enlargement of myo- cytes and development of moderate denaturation necrosis and fibrosis.

This study demonstrated that physiologic status and physical extent of MI can be evaluated using the noninvasive technique of PET. The defect surface area identified with ${ }^{18} \mathrm{~F}-\mathrm{FDG}$ was identical to that identified with PTF, and both agreed well with the results of histology. Furthermore, microscopic degeneration can be characterized as reduced reactivity of quantitative myocardial perfusion to adenosine. The PET-derived PTI, which is known to indicate the absolute fraction of non-scar tissue within the area (22), was reduced not only in the infarcted anterior wall but also in the remote region by $10 \%$. As demonstrated in recent studies (31-34), the reduced PTI in a control region is thought to be caused by the development of microscopic fibrosis.

In this study, left-atrial and LV pressure analyses have not been measured, because the aims of this study were to create a long-survival pig model of MI and global LV dysfunction with remodeling, characterize this model using PET, and compare these parameters with histology before making a complete model of CHF after MI in pigs. One limitation of this study was that, because of technical reasons in our laboratory at the initiation of this study, it was difficult to measure left-atrial and LV pressures.

\section{CONCLUSION}

Our pig model of postinfarction global LV dysfunction was characterized by a high survival rate and large MI, with clear signs of cardiac remodeling, as demonstrated by PET and histology. This animal model might contribute to investigations of MI and new therapies for cardiac remodeling in MI.

\section{ACKNOWLEDGMENTS}

We thank the staff of the Department of Investigative Radiology of National Cardiovascular Center for invaluable technical assistance. This study was supported by a re- 
search grant from New Energy and Industrial Technology Development Organization (NEDO), Japan, and grants for translational research and nanomedicine from the Ministry of Health, Labor and Welfare (MHLW), Japan.

\section{REFERENCES}

1. Saito I, Folsom AR, Aono H, Ozawa H, Ikebe T, Yamashita T. Comparison of fatal coronary heart disease occurrence based on population surveys in Japan and the USA. Int J Epidemiol. 2000;29:837-844.

2. Balady GJ, Jette D, Scheer J, Downing J. Changes in exercise capacity following cardiac rehabilitation in patients stratified according to age and gender: results of the Massachusetts Association of Cardiovascular and Pulmonary Rehabilitation Multicenter Database. J Cardiopulm Rehabil. 1996;16:38-46.

3. Krum H, Haas SJ, Eichhorn E, et al. Prognostic benefit of beta-blockers in patients not receiving ACE-inhibitors. Eur Heart J. 2005;26:2154-2158.

4. Anversa P, Leri A, Kajstura J. Cardiac regeneration. J Am Coll Cardiol. 2006;47:1769-1776.

5. Gheeraert PJ, Henriques JP, De Buyzere ML, De Pauw M, Taeymans Y, Zijlstra F. Preinfarction angina protects against out-of-hospital ventricular fibrillation in patients with acute occlusion of the left coronary artery. J Am Coll Cardiol. 2001;38:1369-1374.

6. Millard RW. Induction of functional coronary collaterals in the swine heart. Basic Res Cardiol. 1981;76:468-473.

7. Roth DM, Maruoka Y, Rogers J, White FC, Longhurst JC, Bloor CM. Development of coronary collateral circulation in left circumflex Ameroid-occluded swine myocardium. Am J Physiol. 1987;253:H1279-H1288.

8. Fallavollita JA, Riegel BJ, Suzuki G, Valeti U, Canty JM Jr. Mechanism of sudden cardiac death in pigs with viable chronically dysfunctional myocardium and ischemic cardiomyopathy. Am J Physiol Heart Circ Physiol. 2005;289:H2688-H2696.

9. Canty JM Jr, Suzuki G, Banas MD, Verheyen F, Borgers M, Fallavollita JA. Hibernating myocardium: chronically adapted to ischemia but vulnerable to sudden death. Circ Res. 2004;94:1142-1149.

10. Fallavollita JA, Canty JM Jr. Differential ${ }^{18} \mathrm{~F}$-2-deoxyglucose uptake in viable dysfunctional myocardium with normal resting perfusion: evidence for chronic stunning in pigs. Circulation. 1999;99:2798-2805.

11. Fallavollita JA, Canty JM Jr. Ischemic cardiomyopathy in pigs with two-vessel occlusion and viable, chronically dysfunctional myocardium. Am J Physiol Heart Circ Physiol. 2002;282:H1370-H1379.

12. Fallavollita JA, Logue M, Canty JM Jr. Stability of hibernating myocardium in pigs with a chronic left anterior descending coronary artery stenosis: absence of progressive fibrosis in the setting of stable reductions in flow, function and coronary flow reserve. J Am Coll Cardiol. 2001;37:1989-1995.

13. Shen YT, Vatner SF. Mechanism of impaired myocardial function during progressive coronary stenosis in conscious pigs: hibernation versus stunning? Circ Res. 1995;76:479-488.

14. Mills I, Fallon JT, Wrenn D, et al. Adaptive responses of coronary circulation and myocardium to chronic reduction in perfusion pressure and flow. Am J Physiol. 1994;266:H447-H457.

15. Roth DM, White FC, Nichols ML, Dobbs SL, Longhurst JC, Bloor CM. Effect of long-term exercise on regional myocardial function and coronary collateral development after gradual coronary artery occlusion in pigs. Circulation. 1990;82:1778-1789.
16. Shen YT, Lynch JJ, Shannon RP, Wiedmann RT. A novel heart failure model induced by sequential coronary artery occlusions and tachycardiac stress in awake pigs. Am J Physiol. 1999;277:H388-H398.

17. Guide for the Care and Use of Laboratory Animals. Washington, DC: National Academy Press; 1996.

18. O'Konski MS, White FC, Longhurst J, Roth D, Bloor CM. Ameroid constriction of the proximal left circumflex coronary artery in swine: a model of limited coronary collateral circulation. Am J Cardiovasc Pathol. 1987;1:69-77.

19. Iida H, Takahashi A, Tamura Y, Ono Y, Lammertsma AA. Myocardial blood flow: comparison of oxygen-15-water bolus injection, slow infusion and oxygen15-carbon dioxide slow inhalation. J Nucl Med. 1995;36:78-85.

20. DeFronzo RA, Tobin JD, Andres R. Glucose clamp technique: a method for quantifying insulin secretion and resistance. Am J Physiol. 1979;237:E214-E223.

21. Knuuti MJ, Nuutila P, Ruotsalainen U, et al. Euglycemic hyperinsulinemic clamp and oral glucose load in stimulating myocardial glucose utilization during positron emission tomography. J Nucl Med. 1992;33:1255-1262.

22. Iida H, Tamura Y, Kitamura K, Bloomfield PM, Eberl S, Ono Y. Histochemical correlates of ${ }^{15} \mathrm{O}$-water-perfusable tissue fraction in experimental canine studies of old myocardial infarction. J Nucl Med. 2000;41:1737-1745.

23. Iida $\mathrm{H}$, Rhodes CG, de Silva R, et al. Myocardial tissue fraction: correction for partial volume effects and measure of tissue viability. J Nucl Med. 1991;32: 2169-2175.

24. Iida H, Rhodes CG, de Silva R, et al. Use of the left ventricular time-activity curve as a noninvasive input function in dynamic oxygen-15-water positron emission tomography. J Nucl Med. 1992;33:1669-1677.

25. de Silva R, Yamamoto Y, Rhodes CG, et al. Preoperative prediction of the outcome of coronary revascularization using positron emission tomography. Circulation. 1992;86:1738-1742.

26. Yamamoto Y, de Silva R, Rhodes CG, et al. A new strategy for the assessment of viable myocardium and regional myocardial blood flow using ${ }^{15} \mathrm{O}$-water and dynamic positron emission tomography. Circulation. 1992;86:167-178.

27. Moses MA, Addison PD, Neligan PC, et al. Inducing late phase of infarct protection in skeletal muscle by remote preconditioning: efficacy and mechanism. Am J Physiol Regul Integr Comp Physiol. 2005;289:R1609R1617.

28. Kitakaze M, Node K, Minamino T, et al. Role of activation of protein kinase $\mathrm{C}$ in the infarct size-limiting effect of ischemic preconditioning through activation of ecto-5'-nucleotidase. Circulation. 1996;93:781-791.

29. Kharbanda RK, Mortensen UM, White PA, et al. Transient limb ischemia induces remote ischemic preconditioning in vivo. Circulation. 2002;106:2881-2883.

30. Chen PS, Chen LS, Cao JM, Sharifi B, Karagueuzian HS, Fishbein MC. Sympathetic nerve sprouting, electrical remodeling and the mechanisms of sudden cardiac death. Cardiovasc Res. 2001;50:409-416.

31. Knaapen P, Boellaard R, Gotte MJ, et al. Perfusable tissue index as a potential marker of fibrosis in patients with idiopathic dilated cardiomyopathy. $\mathrm{J} \mathrm{Nucl}$ Med. 2004;45:1299-1304.

32. Knaapen P, Boellaard R, Gotte MJ, et al. The perfusable tissue index: a marker of myocardial viability. J Nucl Cardiol. 2003;10:684-691.

33. Knaapen P, Bondarenko O, Beek AM, et al. Impact of scar on water-perfusable tissue index in chronic ischemic heart disease: evaluation with PET and contrastenhanced MRI. Mol Imaging Biol. 2006;8:245-251.

34. Knaapen P, van Dockum WG, Bondarenko O, et al. Delayed contrast enhancement and perfusable tissue index in hypertrophic cardiomyopathy: comparison between cardiac MRI and PET. J Nucl Med. 2005;46:923-929. 